\title{
Alcune osservazioni su l'idillio Alla luna
}

\author{
Selene Sarteschi
}

Università degli Studi di Pavia

\section{Abstract}

Nell'idillio Alla luna, Giacomo Leopardi trascrive poeticamente i dati della riflessione che ha elaborato nello Zibaldone intorno a quel peculiare e terapeutico carattere della memoria che riesce a scalfire il limite del tempo e del dolore. Così la "graziosa luna», a cui si rivolge il poeta, diventa il correlativo oggettivo di quella condizione emotiva che risorge dal passato al presente e che entra in simbiosi con il tema centrale del componimento, il piacere della «ricordanza».

Parole chiave: luna, malinconia, crepuscolo, memoria, illusioni.

\section{Abstract}

In the idyll Alla luna, Giacomo Leopardi poetically transcribes the details of the reflection in the Zibaldone, on that unusual and therapeutic nature of memory which attenuates the limits of time and unhappiness. The "graziosa luna», which the poet addresses, becomes the correlation of the emotional condition that arises from contemplation of the past and enters into a symbiosis with the with central subject of the poem, i.e. the pleasure of «recollection».

Key words: moon, melancholy, dusk, memory, hopes.

O graziosa luna, io mi rammento che, or volge l'anno, sovra questo colle io venia pien d'angoscia a rimirarti: e tu pendevi allor su quella selva siccome or fai, che tutta la rischiari.

Ma nebuloso e tremulo dal pianto che mi sorgea sul ciglio, alle mie luci il tuo volto apparia, che travagliosa era mia vita: ed è, né cangia stile, o mia diletta luna. E pur mi giova la ricordanza, e il noverar l'etate del mio dolore. Oh come grato occorre 
nel tempo giovanil, quando ancor lungo la speme e breve ha la memoria il corso, il rimembrar delle passate cose, ancor che triste, e che l'affanno duri!

L'idillio Alla luna che, nell'ordinamento definitivo dei Canti $^{1}$ occupa la XIV posizione, fu composto a Recanati «con tutta probabilità nel 1820, forse nel luglio. Fu pubblicato la prima volta, con gli altri idilli (L'infinito, La sera del dì di festa, Il sogno, La vita solitaria e il Frammento XXXVII), nel "Nuovo Ricoglitore» di Milano (gennaio 1826) e, subito dopo, nei Versi (Bologna 1826) col titolo La ricordanza. Accolto nella prima edizione dei Canti (Firenze 1831) col titolo definitivo». ${ }^{2}$

Mentre il poeta si impegna nell'impresa delle due prime canzoni civili e politiche, All'Italia, Sopra il monumento di Dante e, probabilmente, se accettiamo per la datazione di Alla luna il luglio 1820, anche della complessa stesura della canzone Ad Angelo Mai, egli in contemporanea porta avanti una ben diversa poetica, brevemente sintetizzata con queste parole: «Idilli esprimenti situazioni, affezioni, avventure storiche del mio animo». ${ }^{3}$ In primo piano —negli Idilli- è dunque l'avventura dell' io', con il corredo dei suoi affetti e l'implicita premura di inquadrarli, per quanto soggettivamente, in una situazione reale. Tanto più reale quanto maggiore si presenta la necessità dell'ideale viaggio conoscitivo che il poeta compie in se stesso, anche alla ricerca delle ragioni di una sensibilità che, già all'altezza del 1819-20, si riconosce vulnerata da un'acutissima percezione della malinconia, parola chiave nella visione del mondo leopardiana che il poeta diciottenne identifica con chiarezza come suo proprio male in una lettera a Pietro Giordani (35) del 1817: ${ }^{4}$

1. Per un inquadramento generale dell'opera leopardiana si rimanda all'utile monografia di Gino Tellini, Leopardi, Roma: Salerno Editrice, 2001. La bibliografia dedicata al poeta è [quasi] immensa e ci si limita qui a indicare unicamente i contributi che abbiamo tenuto presenti in queste pagine, per cui cfr. Sebastiano TIMPANARO, Classicismo e illuminismo nell'Ottocento italiano, Pisa: Nistri-Lischi, 19692 [1965]; Marco SANTAGATA, Quella celeste naturalezza. Le canzoni e gli idilli di Leopardi, Bologna: Il Mulino, 1994; Luigi BLASUCCI, Leopardi e i segnali dell'infinito, Bologna: Il Mulino, 1985; ID., I tempi dei "Canti», nuovi studi leopardiani, Torino: Einaudi, 1996; ID., Lo stormire del vento tra le piante. Testi e percorsi leopardiani, Venezia: Marsilio, 2003.

2. Cfr. Giacomo LeOpardi, Canti, a cura di Niccolò Gallo e Cesare Garboli, Torino: Einaudi, 1993, p. 113. Per le citazioni ci riferiremo sempre a tale edizione. Per quanto concerne la data di composizione della poesia diversa è l’opinione di Luigi Blasucci: «La celebrazione di un "piacere dell'immaginazione» accomuna Linfinito all'altrettanto breve (sedici versi) e coevo (1819) Alla luna, anche se nel libro dei Canti i due componimenti risultano separati dalla posteriore Sera del dì di festa, per un principio di alternanza tra idilli brevi e lunghi». Cfr. Luigi BlasuCCI, I tempi dei "Canti», Nuovi studi leopardiani, op. cit., p. 194.

3. Tutte le opere (TO), vol. I, Firenze: Sansoni, 1969, vol. I, p. 372. (Pubblicazione degli scritti leopardiani con introduzione a cura di Walter Binni, con la collaborazione di Enrico Ghidetti.)

4. Cfr. Giacomo LeOPARd, Epistolario, a cura di Francesco MoronCini e Giovanni FerreTTI, Firenze: Le Monnier, 1934-1941. Ma la stessa lettera, del 30 aprile 1817 (n. 32), si può leggere in TO, op. cit., p. 1025. 
[...] ed io di dieciott'anni potrò dire, in questa caverna [scil. Recanati] vivrò e morrò dove sono nato? Le pare che questi desideri si possano frenare? che siano ingiusti soverchi sterminati? che sia pazzia il non contentarsi di non veder nulla, il non contentarsi di Recanati? [...] A tutto questo aggiunga l'ostinata nera orrenda barbara malinconia che mi lima e mi divora, e collo studio s'alimenta e senza studio s'accresce. (Sempre miei i corsivi se non specificato diversamente.)

Ma, ai fini dell'idillio Alla luna, è illuminante ciò che Leopardi aggiunge poco dopo, nella medesima lettera:

So ben io qual è, e l'ho provata, ma ora non la provo più, quella dolce malinconia che partorisce le belle cose, più dolce dell'allegria, la quale, se mee permesso di dir così, è come il crepuscolo, dove questa è notte fittissima e orribile, è veleno, come Ella dice, che distrugge le forze del corpo e dello spirito.

La contraddizione che si annida nella «malinconia più dolce dell'allegria» non è ancora un ossimoro, ma è l'esatta descrizione di colui che emblematizzerà, nella contraddizione, una delle sue cifre poetiche perché nella antinomia esperimenta l'oscillante natura delle proprie pulsioni, i polivalenti e molteplici «moti del cuore»" ${ }^{5}$ umano. L'inquietudine giovanile, percorsa da brividi intensissimi di felicità, diventerà, nel tempo, qualcosa di molto diverso e non è questa la sede per affrontare un tema tanto vasto: basti un accenno a quanto di sentimentalmente, al contempo costante e variabile, nello spazio di un manipolo di endecasillabi — precisamente 14 sino all'aggiunta dei versi 13-14 operata da Leopardi sull'esemplare Starita dei Canti (Napoli 1835) e che vide la luce per la prima volta nel libro curato dal Ranieri (Firenze 1845)_ è possibile ritrovare, in questo idillio, come prova di quella lucidità intellettiva che nasce e si esprime (manifestandosi in un interrotto e sistematico circuito psichico che congiunge affettività e razionalità) attraverso la personale analisi emotiva, la quale giunge a sfociare nella compensazione gratificante e consolatoria della poesia:

E lo stesso spettacolo della nullità, è una cosa in queste opere [sc. di genio], che par che ingrandisca l'anima del lettore, la innalzi, e la soddisfaccia di se stessa e della propria disperazione. (Gran cosa, e certa madre di piacere e di entusiasmo, e magistrale effetto della poesia, quando giunge a fare che il lettore acquisti maggior concetto di se, e delle sue disgrazie, e del suo stesso abbattimento e annichilamento di spirito). ${ }^{6}$ (Zibaldone, 3...4 Ott. 1820).

5. Cfr. Il Risorgimento, vv. 1-6: «Credei ch'al tutto fossero / in me, sul fior degli anni, / mancati i dolci affanni / della mia prima età: / i dolci affanni, i teneri / moti del cor profondo"; Le ricordanze, vv. 170-73: «[...] e fia compagna / d'ogni mio vago immaginar, di tutti / i miei teneri sensi, i tristi e cari / moti del cor, la rimembranza acerba».

6. Giacomo LeOPARDI, Zibaldone di pensieri, a cura di Giuseppe PACELla, Milano: Garzanti, 1991, p. 260 (le citazione dello Zibaldone si riferiscono sempre a questa edizione). 
Come osserva magistralmente Blasucci «sul piano dell'esecuzione poetica, un decisivo elemento di distinzione [di Alla luna] rispetto all' Infinito è dato dall'impostazione della «voce»: alla pura dizione interiore di quell'idillio si sostituisce qui la forma del colloquio, avviata dal vocativo «O graziosa luna» e proseguita con movimenti affabili e discorsivi per il resto della lirica [...]».7

Ripensando al passo della lettera al Giordani appena rievocato, nel luogo in cui Leopardi richiama l'immagine della "dolce malinconia» paragonandola metaforicamente al «crepuscolo», è facile cogliere il legame fra lo stato d'animo espresso nel testo e lo spettacolo naturale che a quello stato d'animo ha dato origine: il colle, la selva, soprattutto la luna che, nel caso specifico, rappresenta il «tu» di un discorso silenzioso ma intenso, di un dialogo in cui il corpo celeste - come attraverso la sua luce espansiva è in grado di illuminare la terra- può fare altrettanto nell'animo, rischiarando la malinconia, sviandola letteralmente e metaforicamente dalla "notte» al "crepuscolo»: e innestando così il processo emotivo che dalla disforia si muoverà verso il «giovamento» della «rimembranza». Il medesimo concetto espresso nell'epistola al Giordani ritorna nel seguente brano dello Zibaldone: "Ma quantunque chi non ha provato la sventura non sappia nulla, è certo che l'immaginazione e anche la sensibilità malinconica non ha forza senza un'aura di prosperità, e senza un vigor d'animo che non può stare senza un crepuscolo un raggio un barlume di allegrezza» (24 Giu. 1820). ${ }^{8}$

Questo brano dello Zibaldone, risalente al giugno 1820, si adatta in modo perfetto alla «situazione» contingente, all' «avventura storica» dell' io' poetico che l'idillio descrive: la "graziosa luna» è il correlativo oggettivo di quella condizione emotiva che risorge dal passato al presente — «or volge l'anno»— e che entra in simbiosi con il tema centrale del componimento, il piacere della «ricordanza». Nell'incipit, subito dopo il vocativo alla luna che occupa la prima parte dell'endecasillabo, l'«io» è collocato in posizione centrale e comunque iniziale del secondo emistichio: «io mi rammento». Inizia da subito il percorso della memoria, con un verbo — «rammentare»— hàpax nei Canti.

L'endecasillabo incipitario possiede una propria autonomia semantica: il lettore può comprendere il significato compiuto del verso senza bisogno di integrazioni. Il tenue enjambement fra il primo e il secondo verso rappresentato dal "che» congiuntivo è, dunque, il necessario slittamento, la lievissima inarcatura sintattico-grammaticale atta a portare avanti il discorso: dopo il volgersi di un anno, al probabile scadere di un anniversario, nello stesso luogo e con il medesimo stato d'animo, il poeta si indirizza di nuovo alla "graziosa luna»? (e si noti

7. Luigi BlaSUCCI, op. cit., p. 194.

8. Giacomo LeOPARDI, Zibaldone di pensieri, op. cit, p. 136.

9. Alla luna personificata come figura femminile (cfr. v. 8) Leopardi attribuisce un aggettivo che - in riferimento ad una persona fisica — spetterà, nei suoi scritti, solo a Fanny Targioni Tozzetti: «Addio, bella e graziosa Fanny. Appena ardisco pregarvi di comandarmi, sapendo che non posso nulla. [...] e credetemi sempre vostro Leopardi». Giacomo LEOPARDI, Epistolario, a c. di Francesco MORONCINI e Giovanni FerReTTI, op. cit., lettera 840 (1832).3. Nell'edizione di TO, cfr. lettera 831 A Fanny Targioni Tozzetti - Livorno, Firenze 16 agosto [1832], p. 1389. 
che anche questo aggettivo è hàpax nei Canti): tornando esplicitamente a «rimirarla». Una rapida ricerca elettronica ci consente di affermare che il verbo «rimirare» compare, in Leopardi, nelle forme seguenti: «rimira» (una volta; $\mathrm{La}$ tempesta, 34); «rimirai» (due volte; Le rimembranze, 77 e 100); «rimirando» (due volte; Appressamento, canto 1.22; Paralipomeni alla Batracomiomachia, canto 2.12); «rimirar» (tre volte; L'amicizia, 133, Re Magi 2.76, Da Ovidio, 18); «rimirarmi» (una volta; Da Ovidio, 36); «rimirava» (una volta; Le rimembranze, 17)); «rimirò» (una volta; Paralipomeni, canto 7.36), mentre la forma «rimirarti» occorre solo due volte: ne L'amicizia (147) che appartiene alle Poesie degli anni $1809-1810^{10} \mathrm{e}$, nei Canti, in Alla luna. ${ }^{11}$

A questo punto, attraverso le informazioni contenute nei primi tre versi, già sappiamo che la memoria ha recuperato un'emozione antica e che, nonostante il trascorrere del tempo, il paesaggio è rimasto immutato: «sovra questo colle» è ancora e sempre il medesimo rifugio dell'anno precedente, quasi con certezza "questermo colle» evocato ne Linfinito (v. 1). E i due sintagmi, «questo colle»-«quest'ermo colle», che il lettore accosta in una lettura sincronica di entrambi i testi, creano un'atmosfera di intimità e di affabile ripetitività, sottolineata dall'aggettivo dimostrativo che, in Alla Luna, si accoppia parallelamente, come nel primo idillio, all'altro aggettivo dimostrativo "quella»:

Sempre caro mi fu quest'ermo colle, e questa siepe, che da tanta parte dell'ultimo orizzonte il guardo esclude.

Ma sedendo e mirando, interminati spazi di là da quella, [...]. (L'infinito, vv. 1-5)

O graziosa luna, io mi rammento che, or volge l'anno, sovra questo colle io venia pien d'angoscia a rimirarti: e tu pendevi allor su quella selva, siccome or fai, che tutta la rischiari. (Alla luna, vv. 1-5).

Sempre sul monte Tabor, nei pressi di Recanati, sullo sfondo di una ciclica ricorrenza cronologica, il protagonista si reca nel medesimo luogo dove scatta il procedimento mentale che crea la sensazione del ricordo, del quale, a sua volta, il poeta — misurando in particolare le minime diffrazioni emotive fra il momento trascorso e quello attuale - offre la descrizione precisa seguendo i movimenti temporali e interiori che, dal passato, si ricongiungono al presen-

10. Cfr. Maria CORTI (a cura di), Entro dipinta gabbia. Tutti gli scritti inediti, rari e editi 18091810 di G. Leopardi, Milano: Bompiani, 1972: «Vieni al mio sen, diletto amico, alfine / rimirarti poss'io; l'estremo amplesso / da un compagno fedel ricevi [...]».

11. Il verbo «mirare», topico del linguaggio lirico due-trecentesco, nel significato di «contemplare», è usato frequentemente anche nella forma prefissata «rimirare», con il medesimo valore semantico. In Leopardi riassume la pregnante valenza semantica stilnovistica: quella dell'ammirata ed incantata contemplazione dell'oggetto amato, in questo caso la luna femminilmente personificata. 
te: e la luna che "pende» dal cielo (e Leopardi recupera, con l'uso di tale verbo, anche l'idea del costante cammino pendolare del globo celeste) si presenta, lo abbiamo visto, come il dato oggettivo e concreto che favorisce il passaggio soggettivo dall'angoscia al rasserenamento della «ricordanza». I primi cinque versi dell'idillio costruiscono un vero e proprio quadro che incornicia il componimento dove si svolge l'avventura dell' 'io'. Il punto fermo, a chiusura del quinto endecasillabo, impone una sosta sì che la scena si visualizzi nei suoi contorni, pronta ad accogliere il proseguimento del pensiero.

Si può osservare che dal verso 6 la storia ha una sorta di «ricominciamento", agevolato da un elemento stilistico-grammaticale carissimo a Leopardi, la congiunzione avversativa «Ma»:
Ma nebuloso e tremulo dal pianto
che mi sorgea sul ciglio, alle mie luci
il tuo volto apparia, che travagliosa
era mia vita: ed è, né cangia stile,
o mia diletta luna. [...] (Alla luna, vv. 6-10).

Anche ne Linfinito la congiunzione avversativa compare (v. 4) — "Ma sedendo e mirando, interminati / spazi di là da quella [...]»— ed in entrambi i componimenti essa ha la funzione di connotare un passaggio e una diversificazione semantica (più o meno marcata) ${ }^{12}$ rispetto «al già detto». E, in Alla luna, infatti, Leopardi rammenta «ora», nel momento presente, che «allora» (l'anno prima) il "volto» lunare appariva ai suoi occhi «nebuloso e tremulo» (anche questi aggettivi nella forma maschile e singolare sono hàpax nei Canti): sfocato a causa del "pianto» che «sorgendo» «sul ciglio» diminuiva allo sguardo la nitidezza dello splendore lunare che invece, al contrario (per cui il «Ma»), si espandeva ovunque, in tutto il suo nitore, sul paesaggio circostante. E risperimentando quel senso di lontana, a sua volta tremula ed indeterminata vaghezza della visione, adesso nitida, il poeta può, con maggiore distacco e obiettività, decifrare la sensazione antica connessa alla rimembranza, tanto più poetica e consolatoria (complice l'attuale scadere di un anniversario ${ }^{13}$ alla vista interiore che percepisce ancora una volta il piacere del vago e dell'indefinito di un tempo:

Le descrizioni della notte ec. sono poeticissime, perché la notte confondendo gli oggetti, l'animo ne concepisce che un'immagine vaga, indistinta, incompleta, sì di essa, che quanto ella contiene. (Zibaldone, 28 settembre 1821; p. 1798).

12. Il «Ma» de Linfinito, rispetto a quello di Alla luna, possiede indubbiamente uno spessore semantico assai più netto che corrisponde, si può dire, al maggior «peso» poetico dell'idillio. Credo, comunque, che la particella avversativa rivesta una funzione importante anche nella nostra lirica.

13. Giacomo LeOPARDI (con introduzione e commento di M. Fubini. Edizione rifatta con la collaborazione di E. Bigi, II edizione nuovamente riveduta e accresciuta), Canti, Torino: Loescher, 1980. Scrive Fubini: "[l'idillio] fu composto molto probabilmente nel 1819; non sappiamo se prima o dopo l'Infinito. Poiché nel canto si allude ad un anniversario (v. 2, «or volge l'anno"), si può ritenere assai probabile che esso sia stato concepito dal poeta nel giorno del suo compleanno: nell'anno precedente, in quel giorno, aveva gettato sulla carta un 
È pure una bella illusione quella degli anniversari [...] e ci par veramente che quelle tali cose che son morte per sempre né possono più tornare, tuttavia rivivano e sieno presenti come in ombra, cosa che ci consola infinitamente allontanandoci l'idea della distruzione e annullamento che tanto ci ripugna, o illudendoci sulla presenza di quelle cose che vorremmo presenti effettivamente, o di cui pur ci piace di ricordarci per qualche speciale circostanza [...]. Così negli anniversari. (Zibaldone, Dic. 1818...8 Gen. $1820 ;$ p. 60)

Ed è in tale situazione altamente poetica un cui si innesta la memoria del dolore provato, il ricordo del velo delle lacrime sui propri occhi prigionieri di una luce sfocata, in cui si sovrappone la presa di coscienza di un'ossimorica, confortante e tormentata, continuità della sofferenza: «[...] che travagliosa / era mia vita: ed è, né cangia stile, / o mia diletta luna. E pur mi giova / la ricordanza, e il noverar l'etate / del mio dolore [...]» (ivi, vv. 8-12).

In pendant con il primo emistichio dell'incipit, circolarmente, la prima parte del verso 10 — in cui cade la forte e paradigmatica cesura del punto fermo - ripete l'allocuzione alla luna, questa volta accompagnata dall'aggettivo possessivo "mia" che rincalza il valore familiare e tenero dell'espressione. Proprio la luna, "graziosa» e "diletta», è la silenziosa deuteragonista dell'idillio, forma luminosa che assume aspetto umano in una lirica dove la figura retorica della personificazione presentifica e visualizza la densa introspezione che ispira il giovane Leopardi, attestata poeticamente con soave mestizia: quella che ha origine dalla "dolce malinconia che partorisce le cose belle». E dall'analisi interiore nasce la constatazione che rappresenta e rappresenterà un manifesto poetico: la piacevolezza, il giovamento del trascorrere del dolore e del suo ricordo, nonostante il suo perdurare. Lo Zibaldone $\grave{e}$, anche in questo caso, la miniera di quelle annotazioni senza le quali non potremmo apprezzare completamente la profondità dell'ideologia leopardiana:

Per la copia e la vivezza ec. delle rimembranze sono piacevolissime e poeticissime tutte le immagini che tengono del fanciullesco, e tutto ciò che ce le desta (parole, frasi, poesie, pitture, imitazioni o realtà ec). [...] Siccome le impressioni, così le ricordanze della fanciullezza in qualunque età, sono più vive che quelle di qualunque altra età. E sono piacevoli per la loro vivezza, anche le ricordanze d'immagini e di cose che nella fanciullezza erano dolorose, o spaventose ec. E per la stessa ragione ci è piacevole nella vita anche la ricordanza dolorosa, e quando ben la cagion del dolor non sia passata, e quando pure la ricordanza lo cagioni o l'accresca [...]. (Zibaldone, 25 Ott. 1821; p. 1987).

abbozzo di elegia [...]: «Oggi finisco il ventesim'anno. Misero me che ho fatto? Ancor nessun fatto grande. Torpido giaccio fra le mura paterne», ecc. (cfr. Poesie e prose, I, p. 381). Il giorno per lui solenne invita il poeta a rivolgersi sul suo passato, a confrontare il dolore di ieri con quello di oggi, a «noverar l'etate del suo dolore», p. 122. 
Chiosa pertinentissima alla parte finale del componimento quando si espungano i versi 13-14, aggiunti dal poeta di proprio pugno sull'esemplare Starita dell'edizione napoletana dei Canti del 1835, quando il suo pessimismo, affilato come rasoio, aveva amputato gran parte della «teoria del piacere»:

\author{
[...] Oh come grato occorre, \\ nel tempo giovanil, quando ancor lungo \\ la speme e breve ha la memoria il corso, \\ il rimembrar delle passate cose, \\ ancor che triste, e che l'affanno duri! (vv. 12-16)
}

Un altro brano dello Zibaldone costituisce la prova che il giovane Leopardi - siamo nel 1821 - credeva ancora nel potere consolatorio che speranza e illusioni esercitano sul disinganno e sulle "piaghe dell'animo»:

E massimamente è difficile e strano che il dolor d'animo, una sventura non corporale ec. cagionino morte o malattia lungo tempo dopo nato, o avvenuta la detta sventura ec. e che in somma la vita dell'uomo si vada consumando e si spenga a poco a poco per le sole malattie particolari dell'animo. [...] Qual è la cagione? Che il tempo medica tutte le piaghe dell'animo. Ma come? Coll'assuefazione, lo so, e grandemente, ma non già con questa sola. Una gran cagione del detto effetto, è ancora che le illusioni poco stanno a riprender possesso e riconquistare l'animo nostro, anche malgrado noi, e l'uomo (purché viva) torna infallibilmente a sperare quella felicità che aveva disperata; prova quella consolazione che avea creduta e giudicata impossibile; dimentica e discrede quell'acerba verità, che avea poste nella sua mente altissime radici; $e$ il disinganno più fermo, totale, e ripetuto, e anche giornaliero, non resiste alle forze della natura che richiama gli errori e le speranze. (16 Genn. 1821; p. 513).

Nel nostro idillio, non considerando per un attimo la tarda giunta dei versi 13-14, il poeta è vicino a queste dichiarazioni. Infatti «[...] Oh come grato occorre / [...] / il rimembrar delle passate cose, / ancor che triste, e che l'affanno duri» (vv. 12-16) è una constatazione in cui l'onnipresente pessimismo leopardiano è pur temperato dalla consapevolezza che l'uomo può -in qualche misura, nonostante il dolore- arrivare ad attingere una qual forma di consolazione e di piacere legata al ciclico ritorno delle illusioni. ${ }^{14}$ Tale piacere coincide con la loro rinascita e con la momentanea cessazione della sofferenza provocata, ad esempio, come abbiamo constatato, dal diletto della rievocazione che ricongiunge il passato al presente, in una forma di recupero dell'emozione che la memoria, col suo potere, custodisce, ripristina e vivifica ancora.

14. Cfr. il seguente passo dello Zibaldone: «Il più solido piacere di questa vita è il piacer vano delle illusioni. Io considero le illusioni come cosa in certo modo reale stante ch'elle sono ingredienti essenziali del sistema della natura umana, e date dalla natura a tutti quanti gli uomi$\mathrm{ni}$, in maniera che non è lecito spregiarle come sogni di uno solo, ma propri veramente dell'uomo e voluti dalla natura, e senza cui la nostra vita sarebbe la più misera e barbara cosa ec.» (ivi, Dic. 1818...8 Gen. 1820; p. 51). 
Il punto centrale della riflessione leopardiana nell'idillio Alla luna sembra essere proprio quest'ultimo, ossia la necessità di procedere a ritroso dal presente al passato con la possibilità di rivolgersi nuovamente dal passato al presente: lungo un incessante processo che, nel movimento, nel fluire del «tempo [che] medica tutte le ferite", prospetta alla mente il concetto salvifico della prosecuzione e di una vitale continuità, a dispetto del nichilismo che - nella visione di Leopardi- tende a distruggere il sostegno affettivo dell'idea di infinito.

In Alla luna tale idea di infinito, in modo assai più implicito che nell'omonimo idillio, è pur presente: spia inequivocabile che rivela come il giovane poeta cresciuto studiando i testi sensisti e illuministi presenti nella biblioteca paterna, anelava - romanticamente, nel senso più ampio che si può attribuire a questa tendenza dell'animo- a oltrepassare i limiti troppo angusti del disinganno razionale, tentando perciò di costruire un proprio sistema poetico-filosofico dove le avventure, le affezioni, le situazioni del cuore tendono verso la più limpida libertà emotiva. La libertà emotiva che sola può spiegare le contraddizioni e le antinomie della coscienza. Scrive Leopardi in un appunto dello Zibaldone del 13 dicembre 1821 (p. 2251):
Alla p. 2234. Tutto ciò che è finito, tutto ciò che è ultimo, desta sempre natu- ralmente nell'uomo un sentimento di dolore, e di malinconia. Nel tempo stes- so eccita un sentimento piacevole, e piacevole nel medesimo dolore, e ciò a causa dell'infinità dell'idea che si contiene in queste parole finito, ultimo, ec. (le quali però sono di loro natura, e saranno sempre poeticissime, per usuali e volgari che siano, in qualunque lingua e stile. E tali son pure in qualsivoglia lingua ec. quelle altre parole e idee, che ho notate in vari luoghi, come poeti- che per se, e per l'infinità che essenzialmente contengono) (13 Dic. 1821). V. p. 2451. (corsivi nel testo).

Anche qui la riflessione si appunta sul concetto che ciò che termina genera malinconia e, al contempo, piacere nella sofferenza: poiché solo quanto è considerato "finito" e "ultimo" può contenere e rendere reale, per contrapposizione logica, l'«infinito» e la sua idea correlata, di cui l'essere umano ha bisogno per fuggire dal dolore e tornarvi, nell'incessante richiesta del piacere: che esiste realmente anche se l'uomo raramente lo prova e, in particolare, nell'istante in cui sta per perdere ciò che ama di più. E questo amore, nell'idillio Alla luna, è l'affezione profonda per la memoria incantata che — dal passato al presente- ricongiunge quanto è trascorso a quanto sta trascorrendo: sia pure questo "quanto» la ricordanza del pianto. Vi è la luna lassù, che pende nel cielo, silente presenza che assiste, nella luce che espande infinita, alla «chiarificazione» e al rasserenamento dell'animo. Ed è lei, la "graziosa» e «diletta luna», in questo breve idillio, a rappresentare il «crepuscolo» della dolce malinconia «delle cose belle».

Solo la tarda introduzione dei versi 13-14 che vorrebbe condurre verso la «notte» il significato o messaggio complessivo dei Canti può considerarsi alla stregua di un approfondimento del materialismo e del cosmico pessimismo. E così è, in effetti. Ma la speranza che, "nel tempo giovanil», ha il compito di 
proiettare il cuore verso un futuro ancor «lungo», mentre la «memoria» ha percorso, e contrario, un cammino ancor "breve», offrono comunque, sino alla fine, la doppia testimonianza di una contraddizione (in cui vive l'inconfondibile leopardiana sete di infinito) e di una coesistenza: quella del disincantato tramonto di un simbolo che si ritrae dinanzi alla «notte» ma che, paradossalmente, continua a riflettere la rimembranza del suo incantato «crepuscolo»:

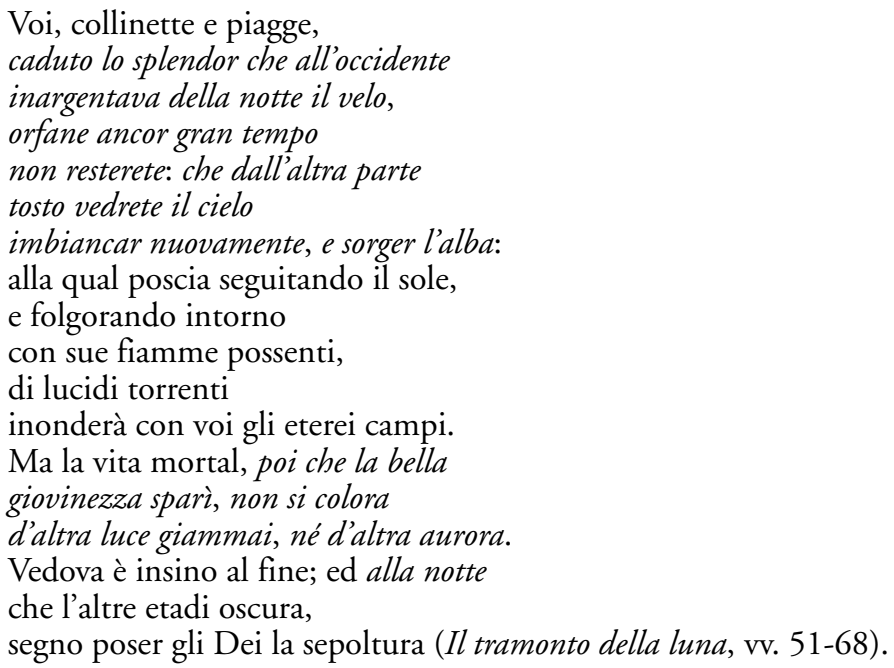

